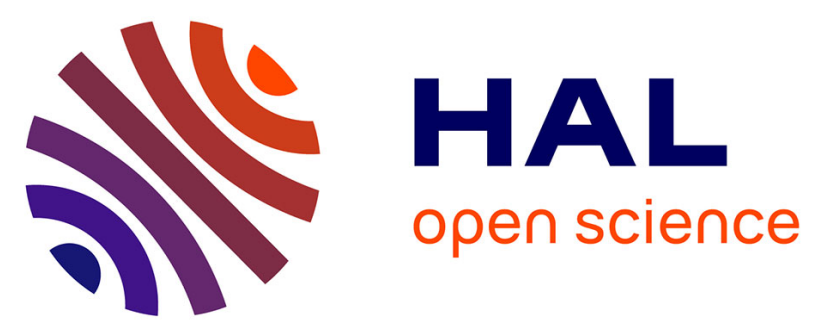

\title{
A multi-faceted strategy to reduce ventilation-associated mortality in brain-injured patients. The BI-VILI project: a nationwide quality improvement project
}

\author{
Karim Asehnoune, Ségolène Mrozek, Pierre François Perrigault, Philippe
}

Seguin, Claire Dahyot-Fizelier, Sigismond S. Lasocki, Anne Pujol, Mathieu

Martin, Russel Chabanne, Laurent Muller, et al.

\section{To cite this version:}

Karim Asehnoune, Ségolène Mrozek, Pierre François Perrigault, Philippe Seguin, Claire DahyotFizelier, et al.. A multi-faceted strategy to reduce ventilation-associated mortality in brain-injured patients. The BI-VILI project: a nationwide quality improvement project. Intensive Care Medicine, 2017, 43 (7), pp.957 - 970. 10.1007/s00134-017-4764-6 . hal-01775139

\author{
HAL Id: hal-01775139 \\ https://hal.science/hal-01775139
}

Submitted on 11 Dec 2019

HAL is a multi-disciplinary open access archive for the deposit and dissemination of scientific research documents, whether they are published or not. The documents may come from teaching and research institutions in France or abroad, or from public or private research centers.
L'archive ouverte pluridisciplinaire HAL, est destinée au dépôt et à la diffusion de documents scientifiques de niveau recherche, publiés ou non, émanant des établissements d'enseignement et de recherche français ou étrangers, des laboratoires publics ou privés. 


\title{
A multi-faceted strategy to reduce
}

\section{ventilation-associated mortality in brain-injured patients. The BI-VILI project: a nationwide quality improvement project}

Karim Asehnoune ${ }^{1,20^{*}}$, Ségolène Mrozek ${ }^{2}$, Pierre François Perrigault ${ }^{3}$, Philippe Seguin ${ }^{4}$, Claire Dahyot-Fizelier ${ }^{5}$, Sigismond Lasocki ${ }^{6}$, Anne Pujol ${ }^{7}$, Mathieu Martin ${ }^{8}$, Russel Chabanne ${ }^{9}$, Laurent Muller ${ }^{10}$, Jean Luc Hanouz ${ }^{11}$, Emmanuelle Hammad ${ }^{12}$, Bertrand Rozec ${ }^{13}$, Thomas Kerforne ${ }^{14}$, Carole Ichai ${ }^{15}$, Raphael Cinotti ${ }^{1}$, Thomas Geeraerts ${ }^{2}$, Djillali Elaroussi ${ }^{7}$, Paolo Pelosi ${ }^{16}$, Samir Jaber ${ }^{17}$, Marie Dalichampt ${ }^{18}$, Fanny Feuillet ${ }^{19}$, Véronique Sebille ${ }^{18,19}$, Antoine Roquilly' and The BI-VILI study group

\begin{abstract}
Purpose: We assessed outcomes in brain-injured patients after implementation of a multi-faceted approach to reduce respiratory complications in intensive care units.
\end{abstract}

Methods: Prospective nationwide before-after trial. Consecutive adults with acute brain injury requiring mechanical ventilation for $\geq 24 \mathrm{~h}$ in 20 French intensive care units (ICUs) were included. The management of invasive ventilation in brain-injured patients admitted between 1 July 2013 and 31 October 2013 (4 months) was monitored and analysed. After the baseline period (1 November 2013-31 December 2013), ventilator settings and decision to extubate were selected as targets to hasten weaning from invasive ventilation. During the intervention period, low tidal volume ( $\leq 7 \mathrm{ml} / \mathrm{kg}$ ), moderate positive end-expiratory pressure (PEEP, $6-8 \mathrm{~cm} \mathrm{H} \mathrm{H}_{2} \mathrm{O}$ ) and an early extubation protocol were recommended. The primary endpoint was the number of days free of invasive ventilation at day 90 . Comparisons were performed between the two periods and between the compliant and non-compliant groups.

Results: A total of 744 patients from 20 ICUs were included (391 pre-intervention; 353 intervention). No difference in the number of invasive ventilation-free days at day 90 was observed between the two periods [71 (0-80) vs. 67 $(0-80)$ days; $P=0.746]$. Compliance with the complete set of recommendations increased from 8 (2\%) to 52 (15\%) patients after the intervention $(P<0.001)$. At day 90 , the number of invasive ventilation-free days was higher in the 60 (8\%) patients whose care complied with recommendations than in the 684 (92\%) patients whose care deviated from

\footnotetext{
*Correspondence: karim.asehnoune@chu-nantes.fr

${ }^{20}$ Service d'Anesthésie Réanimation, CHU de Nantes, 1 Place Alexis

Ricordeau, 44093 Nantes Cedex 1, France

Full author information is available at the end of the article
}

Take-home message: Lung-protective ventilation (association of tidal volume $\leq 7 \mathrm{ml} / \mathrm{kg}$ with PEEP $6-8 \mathrm{~cm} \mathrm{H}_{2} 0$ ) and standardisation of early extubation seemed to hasten weaning from invasive ventilation and reduce the rate of mortality of brain-injured patients but inconsistent adoption limited their impact. 
recommendations [77 (66-82) and $71(0-80)$ days, respectively; $P=0.03$ ]. The mortality rate was $10 \%$ in the compliant group and $26 \%$ in the non-compliant group $(P=0.023$ ). Both multivariate analysis [hazard ratio $(H R) 1.78,95 \%$ confidence interval $(95 \% \mathrm{Cl}) 1.41-2.26 ; P<0.001$ ] and propensity score-adjusted analysis $(\mathrm{HR} 2.25,95 \% \mathrm{Cl} 1.56-3.26$, $P<0.001)$ revealed that compliance was an independent factor associated with the reduction in the duration of mechanical ventilation.

Conclusions: Adherence to recommendations for low tidal volume, moderate PEEP and early extubation seemed to increase the number of ventilator-free days in brain-injured patients, but inconsistent adoption limited their impact.

Trail registration number: NCT01885507.

Keywords: Ventilator weaning, Brain injuries, Airway extubation, Tidal volume, PEEP

\section{Introduction}

The number of patients requiring mechanical ventilation for neurologic injuries is estimated at over 200,000 per year, which translates to a high societal burden and an estimated average cost of over US $\$ 25$ billion annually [1]. While outcomes of brain-injured patients are primarily driven by the underlying neurologic pathology, both the avoidance and treatment of systemic events have been shown to enhance neurologic recovery [2]. Brain-injured patients frequently experience respiratory complications and delayed extubation, which are associated with poor neurologic evolution and death [3]. Accelerating the liberation of brain-injured patients from mechanical ventilation is therefore warranted, and the implementation of interventions designed to prevent ventilator-induced lung injuries has been proposed $[4,5]$.

While the body of data on mechanical ventilation strategies, weaning and extubation in general in patients is steadily increasing, corresponding data are lacking on brain-injured patients, who are frequently excluded from randomized clinical trials in intensive care units (ICUs). Notably, the association of a low tidal volume and a positive end-expiratory pressure (PEEP), so-called lung-protective ventilation, has been shown to improve outcomes in mechanically ventilated patients with healthy lungs [6] and could be effective in brain-injured patients in whom respiratory failure is the most common organ dysfunction [7]. However, the most recent international guidelines for the management of ventilated brain-injured patients lack recommendations for lung-protective ventilation strategies $[8,9]$, and the implementation of such strategies remains relatively uncommon in European neuro-ICUs [10].

Early liberation from mechanical ventilation has also been proposed to prevent respiratory complications in ICUs. However, delayed extubation, which is associated with pneumonia and death in comatose patients [11], occurs in up to $50 \%$ of brain-injured patients [5]. Implementation of a systematic approach to early extubation has been shown to reduce the rate of respiratory complications [12], but to date this strategy is remains infrequently applied in clinical practice.
A single-centre study conducted by our group found that, as compared to a retrospective control period, implementation of an extubation-readiness bundle improves outcomes of brain-injured patients [5]. Based on these findings, we designed the Brain Injury and Ventilator-Induced Lung Injury (BI-VILI, NCT01885507) trial to confirm prospectively this result in a multi-centre study. This before-after study assessed the effect on patient outcomes 90 days after brain injury of an improvement program consisting of the implementation of evidence-based recommendations for the prevention of respiratory complications and delayed extubation.

\section{Methods}

\section{Ethics statement}

The study protocol was approved by the Institutional Ethical Committee (\#2013-04-06, Nantes, France). Nextof-kin were informed of the study, as were patients as soon as their neurologic status was deemed adequate. Consent was waived given that the project was a collaborative institutional quality improvement initiative applied to all patients.

\section{Population and setting}

The study was performed in 20 ICUs in 18 university hospitals in France. Data pertaining to all patients hospitalised in ICUs and requiring mechanical ventilation for $>24 \mathrm{~h}$ during the treatment of an acute brain injury (traumatic brain injury, subarachnoid haemorrhage, or other) were collected and analysed. Brain injuries were defined as a Glasgow Coma Scale (GCS) score of $\leq 12$ combined with one or more acute processes visualised on computed tomography (CT) (tomodensitometry) scans of the head. Exclusion criteria were the decision to withdraw care during the first $24 \mathrm{~h}$ in the ICU or brain death within the first $24 \mathrm{~h}$.

\section{Quality improvement project}

A before--after study design was used. The before period (the so-called pre-intervention phase) consisted of a 4-month-period (July-October 2013) during which time 
data were prospectively recorded for all consecutive patients who (1) were admitted to participating ICUs and (2) fulfilled the inclusion criteria. The quality improvement project was implemented over a 2-month-period (November-December 2013) during which time no patient data were collected. The after period (the so-called intervention phase) ran from January to April 2014, during which time data were prospectively collected for all consecutive patients admitted to participating ICUs.

\section{Pre-intervention phase}

During the pre-intervention phase, ventilator settings and the decision to extubate were at the discretion of the attending physician. The weaning process is described in the Electronic Supplementary Material (ESM Figs. 2 and 3) and was not modified during the study.

\section{Interphase}

To standarise the educational programme, the coordinating centre organised meetings with all principal investigators of the participating ICUs before each study period. The coordinator provided specific material for each meeting, including information on morbidity and mortality associated with respiratory complications in brain-injured patients.

Principal investigators in association with a medical fellow acted as an implementation team. Physicians, residents, physiotherapists and nurses received formal education and clinical training related to lung-protective ventilation and a systemic approach to early extubation. For lung-protective ventilation, a tidal volume of $\leq 7 \mathrm{ml} / \mathrm{kg}$ of predicted body weight (PBW) and a PEEP of 6-8 $\mathrm{cm} \mathrm{H}_{2} \mathrm{O}$ were stipulated. The recommended respiratory rate was 20 cycles $\mathrm{min}^{-1}$, adjusted to reach normocapnia. Extubation was recommended when the following three criteria were met and carried out within $48 \mathrm{~h}$ at the latest: (1) weaning from ventilation support defined as a successful spontaneous breathing trial (30 min T-tube trial or total pressure support level $<10 \mathrm{~cm} \mathrm{H}_{2} \mathrm{O}$ ), (2) effective cough [13] and (3) GCS score of $\geq 10$ (ESM Fig. 2) [14].

All ICUs were provided with reminders of the proto$\mathrm{col}$, the details of which were attached to the ventilators during the intervention phase (ESM Fig. 1). The general coordinator maintained regular contact with investigators of each center through a mailing list (e-mail: bivili. study@gmail.com).

\section{Intervention phase}

During a 4-month-period, the study protocol consisted of conforming recommended guidelines to the protective ventilation strategy and to adopt a systematic approach to early extubation. Patients were followed up until day 90.

\section{Follow-up}

Local investigators recorded data on the following parameters: demographic characteristics, ventilator settings (closest value to $8.00 \mathrm{am}$, up to day +5 ), gas exchange (closest value to $8.00 \mathrm{am}$, up to day +5 ), acute neurologic complications [intracranial pressure (closest value to $8.00 \mathrm{am}$, up to day +5 ), head tomographic tomodensitometry], pneumonia, acute respiratory distress syndrome (ARDS), duration of mechanical ventilation and hospital stay, Glasgow Outcome Scale (GOS) score at day 90 and mortality at day 90 .

\section{Data collection and quality control}

Data were collected prospectively using a specific website by clinicians or research nurses. Detailed information explaining the instructions for data collection and definitions for outcomes were made available to all investigators prior to the initiation of data collection. For quality assurance purposes, data were electronically checked for uniformity and completeness. Errors or unfilled fields generated queries that were returned to each centre for correction.

\section{Outcomes}

The aims of our interventions were to expedite liberation from ventilation and to improve neurologic recovery. Therefore, the primary outcome was the number of invasive ventilation-free days at day 90 , defined as the number of days between days 1 and 90 that a living patient breathed without invasive mechanical ventilation. Dead patients were ascribed zero invasive ventilation-free days [15]. The secondary outcomes included compliance with the multi-faceted approach, the number of ICU-free days at day 90, the rate of respiratory complications (extubation failure, unplanned extubation, tracheotomy, hospital-acquired pneumonia, ARDS), neurologic recovery at day 90 (GOS) and mortality at day 90 .

\section{Definitions}

Adherence to best practices was considered when (1) the tidal volume at day 1 was $\leq 7 \mathrm{ml} \mathrm{kg}^{-1}$ of PBW; (2) the PEEP at day 1 was $>5 \mathrm{~cm} \mathrm{H}_{2} \mathrm{O}$; (3) extubation was recommended as soon as the three criteria mentioned above (i.e. weaning from mechanical ventilation; GCS $\geq 10$; cough) were fulfilled and carried out within 48 h. Full compliance was considered in patients receiving at day 1 a tidal volume $\leq 7 \mathrm{ml} \mathrm{kg}^{-1}$ of PBW and a PEEP of $>5 \mathrm{~cm}$ $\mathrm{H}_{2} \mathrm{O}$ and who experienced no delay in extubation (ESM Fig. 3).

Hospital-acquired pneumonia was identified based on the presence of any two of the following clinical signs: body temperature of $>38{ }^{\circ} \mathrm{C}$; leukocytosis $>12,000 / \mathrm{ml}$; leukopenia $<4000 / \mathrm{ml}$. Purulent pulmonary secretions 
were associated with the appearance of a new infiltrate or changes in an existing infiltrate on chest X-ray and with a positive bacteriological culture of a respiratory tract sample [16].

Evolution of the brain injuries was assessed by comparing the brain CT scan performed at ICU admission and a second brain CT scan performed $48-72 \mathrm{~h}$ after ICU admission.

\section{Statistical analysis}

In a previous single-centre study [5] we found that braininjured patients had $55 \pm 30$ mechanical ventilator-free days by day 90 . We designed the BI-VILI study to detect an augmentation from $55 \pm 33$ invasive ventilation-free days in the pre-intervention phase to up to $65 \pm 33$ invasive ventilation-free days in the intervention phase [5], with a power of $90 \%$ and a type I error of $5 \%$. This design required the inclusion of 458 patients (229 per phase). To achieve this number, and based on a priori expected rate of inclusion per centre, we calculated that periods of 4 months before and after the intervention ( 8 months in total) and the participation of at least 20 ICUs were required.

The primary analysis involved comparing the number of invasive ventilation-free days at day 90 between the two intervention periods (Wilcoxon test). A Fine and Gray model was then generated to compare the duration of mechanical ventilation between the two periods, considering death as a competing event. Extubation was defined as the principal event, and death before extubation was defined as a competing risk. Three analyses were performed a priori: an unadjusted analysis, an adjusted analysis on variables unbalanced between two periods (backward selection) and a propensity score-adjusted analysis. Using the propensity score, we performed inverse probability of treatment weighting (IPTW) using the propensity score. Stabilised IPTW was calculated by multiplying the inverse probability of treatment weight by the marginal probability of treatment, and the robust sandwich covariance matrix was estimated [17]. To estimate the effect of time trends on overall compliance and on duration of mechanical ventilation, time-series analysis on aggregated data was performed (segmented linear regression with auto-correlated errors). A Cox model was used to compare mortality at day 90 between the two intervention periods.

As a secondary analysis, we planned a priori to use the Fine and Gray model to compare the duration of mechanical ventilation of patients whose care complied with the full set of best practice versus those whose care deviated from best practice. Post hoc, we performed two exploratory analyses (ESM Fig. 3): (1) we considered as fully compliant those patients receiving low tidal volume and moderate PEEP but who died before satisfying the extubation criteria; (2) we considered as compliant patients after exclusion of those who were tracheotomised while receiving low tidal volume and moderate PEEP.

The normality of the variables was tested using a Kolmogorov-Smirnoff test. Continuous data were expressed as the mean \pm standard deviation for parametric data and as the median with the interquartile range (IQR; 25 th -75 th percentiles) for non-parametric data. Categorical data were expressed as numbers and percentages. For demographic characteristics and secondary outcomes, the Chi-square test, Student's $t$ test or Wilcoxon test was used, as appropriate. A $P$ value of $<0.05$ for a two-sided test was considered to be statistically significant. Statistical analysis was performed using SAS statistical software (ver. 9.3; SAS Institute, Cary, NC).

\section{Results}

\section{Population}

Halting recruitment before the end of the study period was not planned in the protocol, and the recruitment was higher than expected, with 744 patients included (391 in the pre-intervention phase, 353 in the intervention phase; ESM Fig. 4). Demographic characteristics upon admission to ICU are listed in Table 1.

\section{Compliance with the multi-faceted approach}

Compliance with recommendations is shown in Table 2 . Rates of compliance in the pre-intervention group with tidal volume and PEEP were 27 and 19\%, respectively, as compared with 49 and $67 \%$ in the intervention group $(P<0.001$ and $P<0.001)$. Mean tidal volume on day 1 decreased from $7.5 \pm 1.2 \mathrm{ml} / \mathrm{kg}$ of PBW to $7.0 \pm 1.0 \mathrm{ml} /$ $\mathrm{kg}$ in the intervention group $(P<0.001)$, and this difference persisted throughout the 5 days of respiratory follow-up (Fig. 1a). The mean PEEP during the 5 first days of mechanical ventilation was higher in the intervention than in the pre-intervention group $(P<0.001$; Fig. 1b). The percentage of early extubation did not differ between the two groups ( 54 vs. $51 \%$; $P=0.54$ ). Compliance with the full set of recommendations increased from eight (2\%) patients to 52 patients $(15 \%)(P<0.001)$, with a significant upward time trend for the rate of overall compliance during the intervention period (Fig. 1c). A significant heterogeneity in the rates of compliance was observed among the participating ICUs (ESM Table 2). A decision of care withdrawal was taken in 162 (22\%) patients, among whom the full assessment of adherence was assessed to be unavailable in two $(0.3 \%)$ patients (ESM Fig. 3). 
Primary outcome: number of invasive ventilation-free days at day 90

The number of invasive ventilation-free days at day 90 was 71 (IQR 0-80) in the pre-intervention group and 67 (IQR $0-80)$ in the intervention group $(P=0.75)$. When death was considered as a competing risk (Fine and Gray model), the intervention did not increase the probability of breathing without invasive ventilation [hazard ratio (HR) 1.08; 95\% confidence interval (CI) $0.92-1.27$; $P=0.33$; Fig. 2a]. In the pre-intervention phase, we detected a downward time trend for the duration of mechanical ventilation $(P<0.001)$, while there was no significant overall trend $(P=0.09)$ and no significant trend during the intervention phase $(P=0.27$; Fig. $2 b)$. Neither the initial condition of the patient nor the severity of brain injury significantly influenced the effect of the recommendations (ESM Fig. 5). By day 90, 131 (33.5\%) patients had died in the pre-intervention group as compared with 106 (30\%) in the intervention group (log-rank test $P=0.30$; Fig. $2 \mathrm{c})$.

\section{Effect of adherence to the multi-faceted approach}

Of the 744 patients enrolled in the study, 60 (8\%) received care with full adherence to recommendations (ESM Table 1). A decision to withdraw care was taken in 157 (23\%) patients whose care involved deviations and in five $(8 \%)$ patients who were fully compliant $(P=0.009)$.

Table 1 Demographic characteristics of the study population $(N=744)$

\begin{tabular}{|c|c|c|c|}
\hline Demographic characteristics & Pre-intervention group ( $n=391 ; 52.5 \%$ ) & Intervention group ( $n=353 ; 47.5 \%)$ & $P$ value \\
\hline Age (years) & $51 \pm 17$ & $53 \pm 17$ & 0.06 \\
\hline Male & $251(64 \%)$ & $215(61 \%)$ & 0.36 \\
\hline Weight (kg) & $74 \pm 16$ & $74 \pm 17$ & 0.96 \\
\hline Height (cm) & $171 \pm 9$ & $170 \pm 10$ & 0.12 \\
\hline SAPS $\|$ & $49 \pm 13$ & $49 \pm 12$ & 0.89 \\
\hline SOFA & $8 \pm 3$ & $8 \pm 3$ & 0.80 \\
\hline \multicolumn{4}{|l|}{ Glasgow coma scale ${ }^{a, b}$} \\
\hline Eye & $1.6 \pm 0.9$ & $1.5 \pm 0.8$ & 0.37 \\
\hline Verb & $1.6 \pm 1.0$ & $1.5 \pm 0.9$ & 0.24 \\
\hline Motor & $3.5 \pm 1.7$ & $3.3 \pm 1.7$ & 0.11 \\
\hline Total & $7 \pm 3$ & $6 \pm 3$ & 0.18 \\
\hline One pupil non-reacted & $106(27 \%)$ & $86(25 \%)$ & 0.44 \\
\hline Initial condition & & & 0.07 \\
\hline Traumatic brain injury & $199(51 \%)$ & $139(39 \%)$ & \\
\hline Sub-arachnoid hemorrhage & $84(21 \%)$ & $93(26 \%)$ & \\
\hline Stroke & $47(13 \%)$ & $53(17 \%)$ & \\
\hline Rupture of vessel malformation & $6(2 \%)$ & $8(3 \%)$ & \\
\hline Others & $55(14 \%)$ & $60(17 \%)$ & \\
\hline \multicolumn{4}{|l|}{ Intracranial pressure monitoring } \\
\hline Traumatic brain injury & $147(74 \%)$ & $109(78 \%)$ & 0.34 \\
\hline Sub-arachnoid hemorrhage & $64(79 \%)$ & $71(76 \%)$ & 0.98 \\
\hline Other & $56(52 \%)$ & $72(59 \%)$ & 0.24 \\
\hline Sedative drugs at day 1 & & & 0.16 \\
\hline Midazolam & $299(82 \%)$ & $258(77 \%)$ & \\
\hline Propofol & $60(17 \%)$ & $73(22 \%)$ & \\
\hline Others & $4(1 \%)$ & $6(2 \%)$ & \\
\hline Opioid at day 1 & & & 0.24 \\
\hline Morphine & $18(5 \%)$ & $11(3 \%)$ & \\
\hline Fentanyl & $67(18 \%)$ & $75(23 \%)$ & \\
\hline Sufentanyl & $252(69 \%)$ & $214(65 \%)$ & \\
\hline Remifentanil & $27(7 \%)$ & $32(10 \%)$ & \\
\hline
\end{tabular}

Data in table are presented as the mean \pm standard deviation (SD) or as a number with the percentage in parenthesis, as appropriate ICU intensive care unit, SAPS // simplified acute physiology score, SOFA sequential organ failure assessment

a Measured by a medical doctor before any injections of sedative or hypnotic drugs 
Table 2 Measures of compliance

\begin{tabular}{|c|c|c|c|}
\hline Measures of compliance & Pre-intervention group ( $n=391 ; 52.5 \%$ ) & Intervention group ( $n=353 ; 47.5 \%)$ & $P$ value \\
\hline \multicolumn{4}{|l|}{ Low tidal volume } \\
\hline \multicolumn{4}{|l|}{ Tidal volume } \\
\hline$\leq 7 \mathrm{ml} \mathrm{kg}^{-1}$ of PBW & $106(27 \%)$ & $174(49 \%)$ & $<0.001$ \\
\hline$>7 \mathrm{ml} \mathrm{kg}^{-1}$ of PBW & $217(55 \%)$ & $122(35 \%)$ & \\
\hline Non applicable ${ }^{a}$ & $68(17 \%)$ & $57(16 \%)$ & \\
\hline \multicolumn{4}{|l|}{ Tidal volume } \\
\hline in $\mathrm{ml} / \mathrm{kg}$ PBW & $7.5 \pm 1.2$ & $7.0 \pm 1.0$ & $<0.001$ \\
\hline in $\mathrm{ml}$ & $486 \pm 80$ & $445 \pm 69$ & $<0.0001$ \\
\hline \multicolumn{4}{|l|}{ Moderate PEEP } \\
\hline $\operatorname{PEEP}[n(\%)]$ & & & $<0.001$ \\
\hline$\leq 5 \mathrm{cmH}_{2} \mathrm{O}$ & $297(76 \%)$ & $101(29 \%)$ & \\
\hline$>5 \mathrm{cmH}_{2} \mathrm{O}$ & $73(19 \%)$ & $237(67 \%)$ & \\
\hline Missing data & $21(5 \%)$ & $15(4 \%)$ & \\
\hline PEEP $($ mean \pm SD) & $5 \pm 1.6$ & $6.1 \pm 1.6$ & $<0.001$ \\
\hline Early extubation ${ }^{b}$ & $171(54 \%)$ & $157(51 \%)$ & 0.54 \\
\hline \multicolumn{4}{|l|}{ Compliance to recommendations } \\
\hline Low tidal volume + moderate PEEP & $14(4 \%)$ & $92(31 \%)$ & $<0.001$ \\
\hline Low tidal volume + early extubation & $30(11 \%)$ & $55(21 \%)$ & 0.003 \\
\hline Moderate PEEP + early extubation & $31(10 \%)$ & $108(37 \%)$ & $<0.001$ \\
\hline Overall compliance ${ }^{c}$ & $8(2 \%)$ & $52(15 \%)$ & $<0.001$ \\
\hline
\end{tabular}

Data in table are presented as the mean \pm SD or as a number with the percentage in parenthesis, as appropriate

PBW Predicted body weight, PEEP positive end-expiratory pressure

${ }^{a}$ Non applicable for patients ventilated with an assist-pressure support during the first $24 \mathrm{~h}$

${ }^{\mathrm{b}}$ Extubation in the first $48 \mathrm{~h}$ after weaning from mechanical ventilation, cough and GCS $\geq 10$, not applicable in 73 patients before and 47 after the intervention (death or tracheotomy)

c Association of a tidal volume: $\leq 7 \mathrm{ml} \mathrm{kg}{ }^{-1}$ of predicted body weight, a PEEP $>5 \mathrm{~cm} \mathrm{H}_{2} \mathrm{O}$ and an early extubation in the same patient

The HR for breathing without assistance at day 90 in patients whose care complied with the entire set of recommendations, as compared with patients with deviations in care, was 1.70 (95\% CI 1.33-2.16; $P<0.001$; Fig. 3a). When we considered as fully compliant those patients receiving low tidal volume and moderate PEEP but who had died before reaching the extubation criteria, the HR for breathing without assistance at day 90 in compliant patients was 1.54 (95\% CI $1.05-2.25 ; P=0.03)$. After excluding tracheotomised patients receiving low tidal volume and moderate PEEP, the HR for breathing without assistance at day 90 in compliant patients was 2.27 (95\% CI 1.56-3.31; $P<0.001)$."

In patients with complete compliance, the number of invasive ventilation-free days at day 90 was 77 (66-82) and the rate of death was $10 \%$, as compared with 71 $(0-80)$ invasive ventilation-free days $(P=0.03)$ and a death rate of $26 \%(P=0.02)$ in patients whose care involved deviations (ESM Fig. 6).

To account for potential confounding factors that may affect the comparison of compliant and non-compliant groups, we performed a propensity score-adjusted analysis and a multivariate analysis. Using a propensity score based on demographic characteristics associated with outcomes (centre, age, GCS score, initial condition, one pupil non-reactive) [18], the HR for breathing without invasive ventilation was 2.25 (95\% CI 1.56-3.26, $P<0.001$ ) in patients whose care was fully compliant. Using a multivariate Fine and Gray model adjusted for variables unbalanced between the two phases $(P<0.10$; Table 1) and for centre and GCS score, overall compliance to the quality improvement program was an independent factor associated with the probability of breathing without assistance (HR 1.78, 95\% CI 1.41-2.26; $P<0.001$; ESM Table S3).

\section{Secondary outcomes}

Secondary outcomes are listed in Table 3.

\section{Neurological evolution}

The time evolution of intracranial pressure before and after the implementation of the multi-faceted approach is shown in ESM Fig. 7. The rates at which patients developed one or more episodes of intracranial hypertension did not differ between the two groups (Table 3; $P=0.60$ ). 

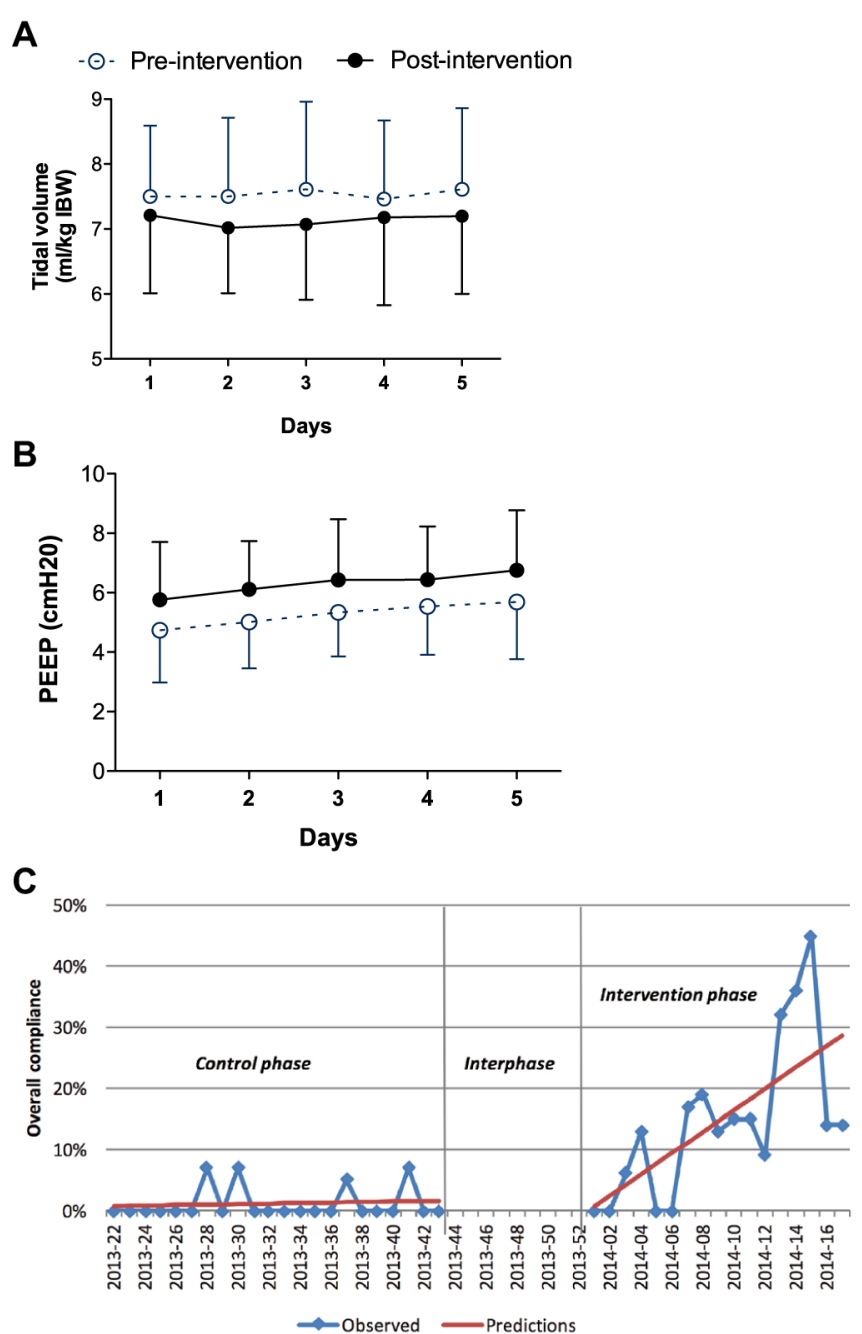

Fig. 1 Compliance to the bundle elements before and during the intervention. Time evolution of tidal volume (a) and positive end-expiratory pressure $(P E E P)(\mathbf{b})$ in the pre-intervention and intervention groups. cTime-series analysis of the overall compliance to the bundle elements ( $y$-axis) by weeks ( $x$-axis) during the pre-intervention and the intervention periods. IBW Ideal body weight

The rate of worsened abnormalities on the brain CT scans was higher in the pre-intervention group than in the intervention group (Table $3 ; P=0.04$ ). The GOS at day 90 did not significantly differ between the two groups (Table $3 ; P=0.40$ ).

\section{Respiratory evolution}

While there was a trend toward lower rates of pneumonia in the intervention group ( 50.4 vs. $44.3 \% ; P=0.10)$, the rate of acute respiratory failure was similar in the pre-intervention and intervention groups ( 26.2 vs. $24.2 \%$; $P=0.53$; Table 3 ). When considering the effects of adherence to the recommendations, the ratio of partial pressure of arterial oxygen and fraction of inspired oxygen $\left(\mathrm{PaO}_{2} / \mathrm{FiO}_{2}\right.$ ratio) was significantly impacted by the tidal volume ( $\leq 7 \mathrm{vs} .>7 \mathrm{ml} / \mathrm{kg}$ of PBW), by the level of PEEP ( $\geq 6$ vs. $<6 \mathrm{~cm} \mathrm{H}_{2} 0$ ) and by the full compliance (vs. patients with any deviations; Fig. 3b-d). The partial pressure of arterial carbon dioxide $\left(\mathrm{PaCO}_{2}\right)$ level was unaffected by the compliance to the multi-faceted approach (Fig. 3b-d).

\section{Discussion}

A multi-faceted approach aiming to implement the combination of lung-protective ventilation and an early extubation strategy did not alter outcomes in the overall population of mechanically ventilated brain-injured patients. No safety issues were detected in terms of neurological tolerance, and evolution of the brain CT scan was improved in the intervention phase. Importantly, the subgroup of patients whose care complied with the entire set of recommendations showed a higher number of invasive ventilation-free days and lower mortality rate at day 90 . 
A

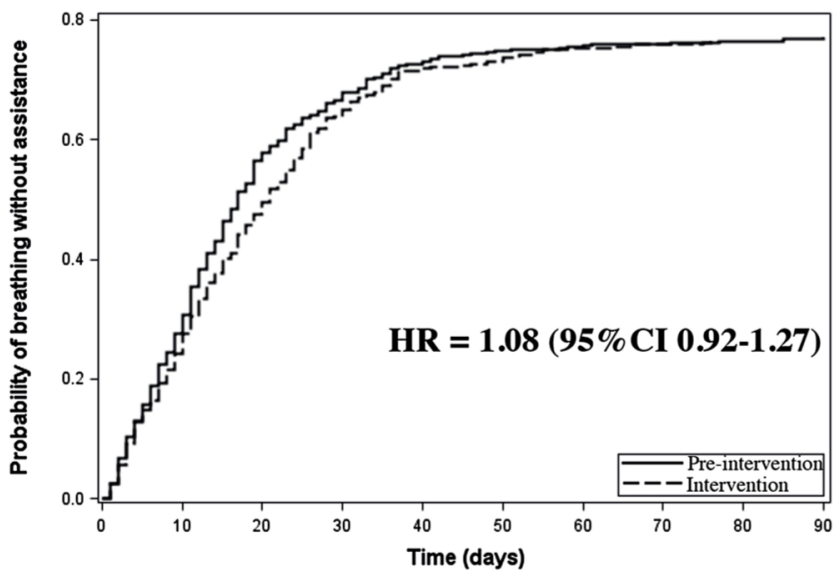

Number of at-risk patients

\begin{tabular}{l|lllllllll|} 
Pre-intervention & 390 & 303 & 220 & 162 & 135 & 130 & 123 & 121 & 119 \\
Intervention & 353 & 244 & 129 & 90 & 67 & 59 & 56 & 55 & 52 \\
\hline
\end{tabular}

B
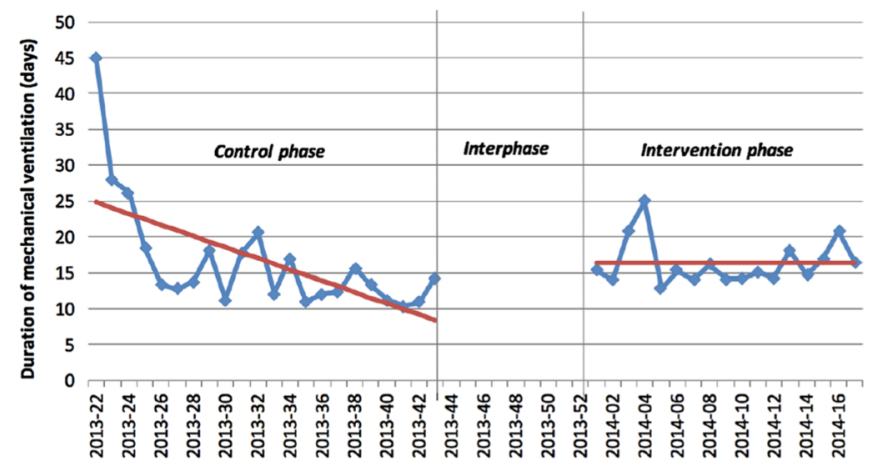

-Observed Predictions

C

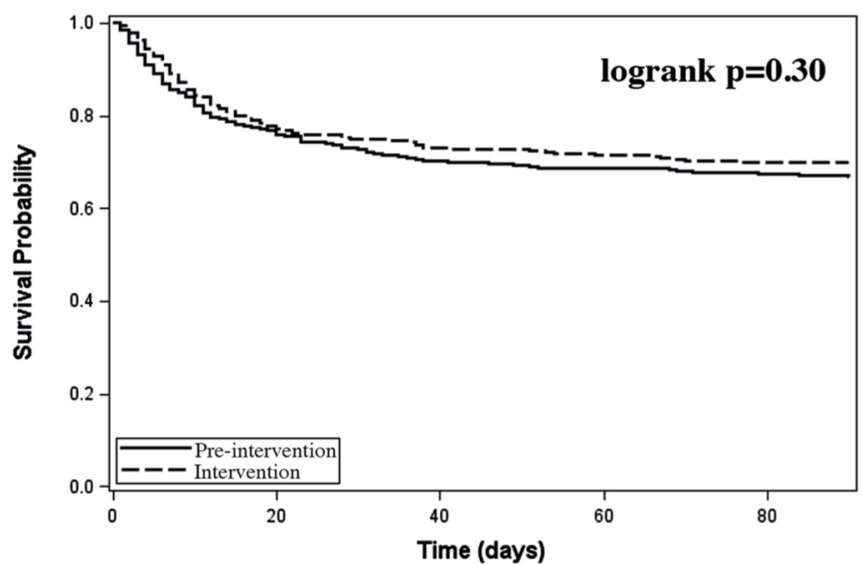

Number of at-risk patients

\begin{tabular}{l|llllllllll|} 
Pre-intervention & 390 & 327 & 299 & 284 & 274 & 270 & 268 & 265 & 263 \\
Intervention & 353 & 302 & 274 & 264 & 258 & 257 & 252 & 249 & 247
\end{tabular}

Fig. 2 Weaning from invasive ventilation and of survival in the pre-intervention and intervention groups. a Cumulative incidence curves for probability of breathing without invasive ventilation in the pre-intervention and intervention groups. $\mathbf{b}$ Time-series analysis of the duration of mechanical ventilation ( $y$-axis) by weeks ( $x$-axis) during the pre-intervention and the intervention periods. $\mathbf{c}$ Probability of survival in the pre-intervention and intervention groups (Kaplan-Meier estimator). HR Hazard ratio, Cl confidence interval 


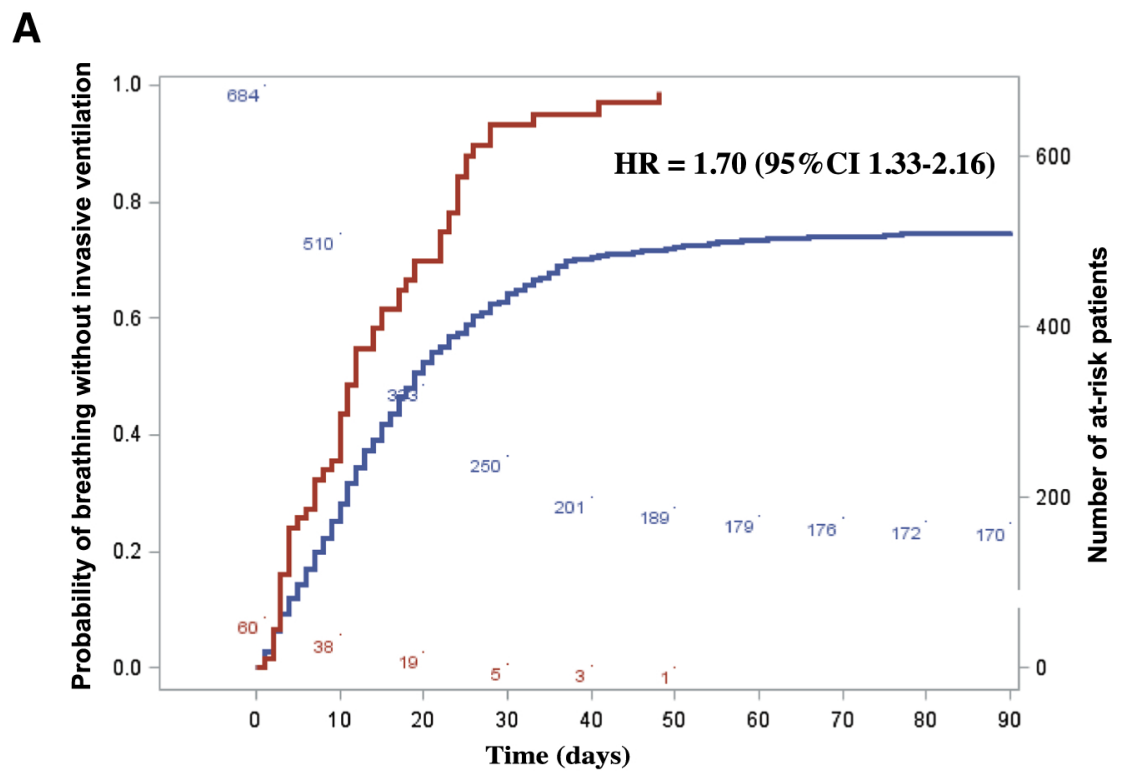

Compliance with the full set of recommendations

Deviation to the recommendations

B

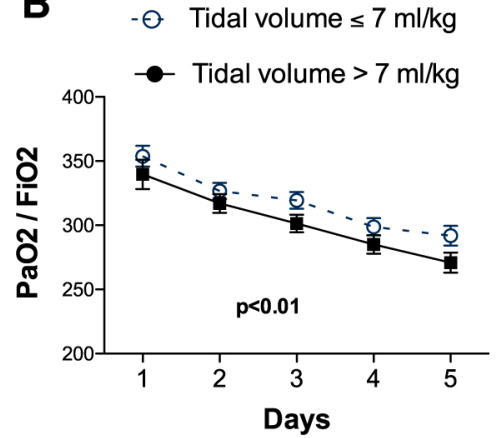

홀

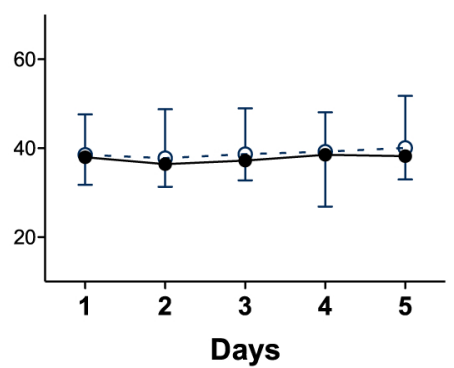

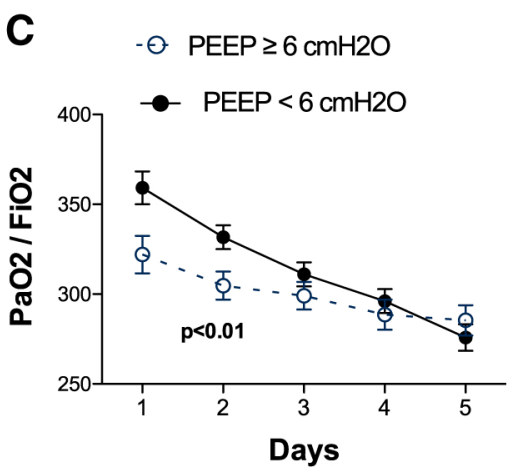

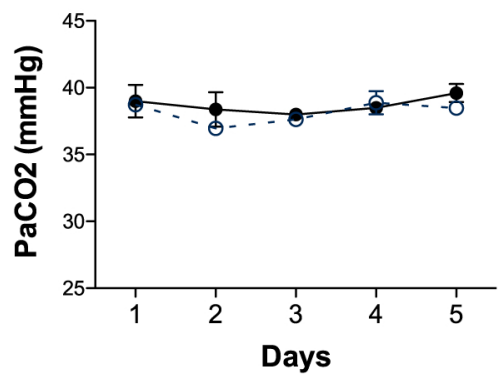

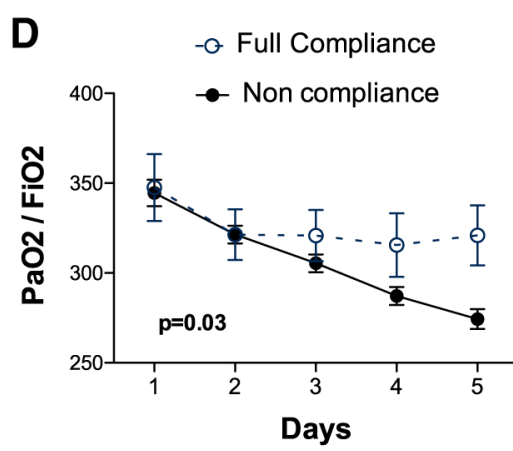

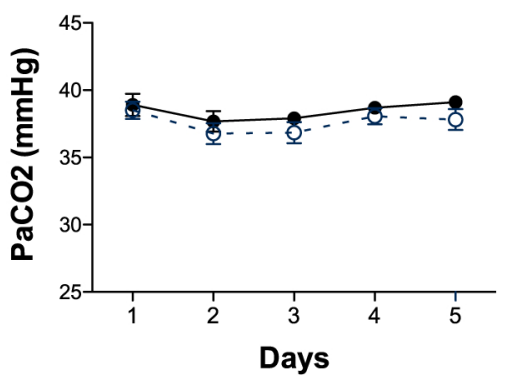

Fig. 3 Effects of adherence to the recommendations. a Cumulative incidence curves for probability of breathing without invasive ventilation in patients whose care complied with the full set of recommendations and for those whose care involved one or two deviations from best practices. b-d Time-course of ratio of partial pressure of arterial oxygen and fraction of inspired oxygen $\left(\mathrm{PaO}_{2} / \mathrm{FiO}_{2}\right)$ and of the partial pressure of arterial carbon dioxide $\left(\mathrm{PaCO}_{2}\right)$ in patients receiving or not a tidal volume of $\leq 7 \mathrm{ml} \mathrm{kg}^{-1}$ of PBW $(\mathbf{b})$, receiving or not a PEEP of $\geq 6 \mathrm{~cm} \mathrm{H}_{2} \mathrm{O}(\mathbf{c})$ or whose care complied with the full set of recommendations and for those whose care involved one or two deviations from best practices (d). $P$ value for the compliance effect, two-way analysis of variance comparisons. Data are presented as the mean \pm standard error of the mean

Ventilation with low tidal volumes improves the outcomes of both critically ill patients with ARDS [19] and patients with healthy lungs $[6,20]$. The choice of $6-8 \mathrm{ml} /$ $\mathrm{kg}$ of PBW as an optimal tidal volume remains a matter of debate [21]. A linear association between the risk of ventilator-induced lung injuries and tidal volumes has been 
Table 3 Secondary outcomes

\begin{tabular}{|c|c|c|c|}
\hline Secondary outcomes & Pre-intervention group ( $n=391 ; 52.5 \%$ ) & Intervention group ( $n=353 ; 47.5 \%$ ) & $P$ value \\
\hline \multicolumn{4}{|l|}{ General outcomes } \\
\hline Invasive ventilation-free days at day 90 & $71[0-80]$ & $67[0-80]$ & $0.75^{\mathrm{b}}$ \\
\hline ICU-free days at day 90 & $62[0-76]$ & $59[0-75]$ & $0.68^{b}$ \\
\hline Death at day 90 & $131(33.5 \%)$ & $106(30.0 \%)$ & $0.30^{c}$ \\
\hline \multicolumn{4}{|l|}{ Neurological outcomes } \\
\hline Intracranial hypertension (>25 mmHg) & $151(42.4 \%)$ & $147(44.4 \%)$ & 0.60 \\
\hline \multicolumn{4}{|l|}{ Medical interventions related to intracranial hypertension } \\
\hline Osmotherapy & $132(36.2 \%)$ & $127(37.7 \%)$ & 0.68 \\
\hline Barbituric & $68(18.6 \%)$ & $57(16.9 \%)$ & 0.55 \\
\hline Moderate hypothermia $\left(33-35^{\circ} \mathrm{C}\right)$ & $37(10.1 \%)$ & $38(11.3 \%)$ & 0.62 \\
\hline Moderate hypocapnia (32-36 mmHg) & $31(8.5 \%)$ & $34(10.1 \%)$ & 0.47 \\
\hline Decompressive craniectomy & $40(11.0 \%)$ & $39(11.6 \%)$ & 0.80 \\
\hline \multicolumn{4}{|c|}{ Evolution of the anomalies on brain CT scan at the 48-72th $\mathrm{h}$} \\
\hline Diminution of the size & $61(18 \%)$ & $69(22.1 \%)$ & 0.03 \\
\hline No modification of the size & $112(33 \%)$ & $119(38.1 \%)$ & 0.04 \\
\hline New or increase in brain-swelling & $75(22.1 \%)$ & $44(14.1 \%)$ & \\
\hline New or increase in bleeding/haematoma & $39(11.5 \%)$ & $44(14.1 \%)$ & \\
\hline Ischaemia & $52(15.3 \%)$ & $36(11.5 \%)$ & \\
\hline $\begin{array}{l}\text { Worsening (brain-swelling, bleeding or ischae- } \\
\text { mia) }\end{array}$ & $166(42.4 \%)$ & $124(35.1 \%)$ & \\
\hline $\begin{array}{l}\text { In-ICU decision of care withdrawal for poor neuro- } \\
\text { logical recovery }\end{array}$ & $89(22.9 \%)$ & $73(20.7 \%)$ & 0.47 \\
\hline Glasgow Outcome Scale at day $90^{a}$ & & & 0.40 \\
\hline Death & $131(38.4 \%)$ & $106(35.3 \%)$ & \\
\hline Persistent vegetative sate & $8(2.4 \%)$ & $5(1.7 \%)$ & \\
\hline Severe disability & $64(18.8 \%)$ & $71(23.7 \%)$ & \\
\hline Moderate disability & $43(12.6 \%)$ & $45(15 \%)$ & \\
\hline Low disability & $95(27.9 \%)$ & $73(24.3 \%)$ & \\
\hline \multicolumn{4}{|l|}{ Respiratory outcomes } \\
\hline \multicolumn{4}{|l|}{ Extubation management, } \\
\hline Extubation failure/Re-intubation & $32(10.4 \%)$ & $38(13.2 \%)$ & 0.28 \\
\hline Unplanned extubation & $30(8.1 \%)$ & $17(5.0 \%)$ & 0.09 \\
\hline \multicolumn{4}{|l|}{ Tracheostomy, } \\
\hline Yes & $52(13.7 \%)$ & $46(13.3 \%)$ & 0.87 \\
\hline Timing (days) & $19[12-30]$ & $19[14-31]$ & 0.66 \\
\hline Hospital-acquired pneumonia & $197(50.4 \%)$ & $156(44.3 \%)$ & 0.10 \\
\hline Acute respiratory distress syndrome & $102(26.2 \%)$ & $85(24.2 \%)$ & 0.53 \\
\hline
\end{tabular}

Data are presented as the median with the interquartile range in square brackets or as the number with the percentage in parenthesis, as appropriate

CT Computed tomography

a Available for 641 patients

b Wilcoxon test

c Log-rank test

demonstrated in brain-injured patients [3, 22-24], and we found that a small difference in tidal volume between the compliant and the non-compliant patients (mean 7.3 and $6.4 \mathrm{ml} / \mathrm{kg}$ of PBW, respectively) was sufficiently large to produce a benefit in best practice-compliant patients. Altogether, these results suggest that lower tidal volumes within $6-8 \mathrm{ml} / \mathrm{kg}$ of PBW are preferable.
Given the theoretical risk of permissive hypercapnia, a major cause of secondary brain damage [25], ventilation of brain-injured patients with low tidal volumes has received little research attention and has been poorly applied in European neuro-ICUs [10]. The present findings confirm that an even lower tidal volume $(7.0 \mathrm{ml} / \mathrm{kg}$ of PBW) can be applied with little change in the levels 
of $\mathrm{PaCO}_{2}$ (Figs. 3b-d). These data are in line with those of a meta-analysis showing that low tidal volumes are associated with a moderate increase in $\mathrm{PaCO}_{2}$ (from 38 to $41 \mathrm{mmHg}$ ) [6]. If $\mathrm{PaCO}_{2}$ levels were deemed inappropriate, the multi-faceted approach used in the present study recommends modification of the respiratory rate as opposed to an increase in tidal volume, but the safety of this strategy needs further investigation. Together with previous findings, our results support the modification of current guidelines for ventilation and more widespread use of low tidal volume in brain-injured patients associated with a close monitoring of $\mathrm{PaCO}_{2}$.

While high PEEP can be beneficial in patients with ARDS [26] and reduce the risk of pneumonia [27], recent multi-centre randomised studies have demonstrated that low tidal volumes [28], but not high PEEP [29], improve outcomes in patients with healthy lungs undergoing abdominal surgery. PEEP-induced increases in intrathoracic pressure may also have deleterious effects, in particular a risk of decreased brain venous drainage, resulting in higher intracranial pressure. However, the effect of PEEP on intracranial pressure has been shown to be small as long as the $\mathrm{PaCO}_{2}$ is controlled [30], and comparison of the two phases revealed no change in the rate of intracranial hypertension. In an exploratory analysis (Fig. 3c), we found that moderate PEEP favourably impacted the time-evolution of the $\mathrm{PaO}_{2} / \mathrm{FiO}_{2}$ ratio in brain-injured patients, leading us to conclude that moderate levels of PEEP can be safely used in brain-injured patients; however, we cannot confirm that a $6-8 \mathrm{~cm} \mathrm{H}_{2} \mathrm{O}$ is the optimal level of PEEP in this population. Other studies are required to address the optimal level of PEEP for invasive ventilation of brain-injured patients.

The multi-faceted approach had no effect on the rate of early extubation, which reached $50 \%$ of participating patients over the course of the study. The high rate of delayed extubation in the intervention phase underscores the difficulties faced by clinicians who must perform extubation in neurologic patients [31]. Indeed, extubation failure is observed at an average rate of $20 \%$ of patients with structural coma on admission and is associated with significant morbidity $[5,13]$. However, failure to achieve early extubation might represent an epiphenomenon occurring as a result of more severe brain injury. In the BI-VILI initiative, we strongly recommended the decision to extubate patients weaned from mechanical ventilation as soon as the GCS was $>10$ in association with cough. The measure of the GCS in mechanically ventilated patients is challenging; thus the addition of other criteria, such as swallowing or visual pursuit [32], might have increased the predictive value of this clinical score, and thus probably enhance the adhesion of clinicians to this recommendation.
The overall adherence to the improvement program was low, and patient care complied with the entire set of best practices only in $15 \%$ of cases after the intervention. This proportion falls within the range reported in a previous before-after study [33] and in routine application of evidence-based recommendations for the prevention of pneumonia [34], management of patients with ARDS [35] or weaning from mechanical ventilation [36]. Several reasons may explain this result. First, the rates of application observed during the pre-intervention period are critical to any interpretation of the efficiency of a quality improvement project. In our study, the extremely small rate of patients receiving the full sets of recommendations before the quality improvement programme (2\%) probably contributed to the low overall compliance in the intervention period. Second, adherence to new evidence in medical practice is a slow process. For example, 8 years after the first Surviving Sepsis Campaign, the overall compliance to the bundles ranges from 19 to $36 \%$ [37]; the proportion of patients receiving the optimal treatment for myocardial infarcts only reached $65 \%$ after a 10-year period of recommendations [38]. In the BI-VILI study, despite an upward trend over time to a higher rate of application after implementation of the quality program, the duration of the intervention period could have been too short ( 4 months) to reach a higher rate of application. Third, our training may have been suboptimal because we did not assess the knowledge of care-givers and trainings were not repeated during the intervention phase $[39,40]$. In conclusion, low adherence is a frequent limit to any quality improvement project, demonstrating the existence of significant barriers to the implementation of recommendations in ICUs. However, before-after studies are informative because they provide information on the applicability of an intervention in clinical practice, thereby helping governing entities to discriminate achievable and non-achievable targets [41]. The development of innovative strategies to hasten the application of recommendations, such as tele-ICU reengineering [42], appears urgently needed.

Another theoretical reason for the failure to adhere to the protocol was our focus on lung complications during the time patients were hospitalised for the treatment of a brain injury. Clinicians may have been afraid that protective ventilation would alter the brain via hypercapnia. Although lung-associated complications are associated with poor outcomes [24], we report here that protective ventilation had no deleterious effect on the brain (no modification of the levels of intracranial pressure was recorded) and even may have enhanced neurological recovery (compliant patients had a higher survival rate). Brain-lung cross-talk should not limit the application of lung-protective strategies [4]. 
Our study has several limitations. First, the results of this before-after study supports an association between the implemented multi-faceted approach and patient outcomes, but they do not demonstrate causation. This design was chosen as it provides a good level of evidence and an accurate estimation of the effect of the intervention [43]. Second, the low rate of application of the recommendations likely reduced the power of the study. Third, the tidal volume was lower in the pre-intervention group than previously reported by our group [5] and others [10], and we observed a downward trend for the duration of mechanical ventilation during the pre-intervention period (Fig. 2b). This unexpectedly low tidal volume in the pre-intervention group can probably be explained by the bias associated with all prospective evaluations of clinician's performance (Hawthorne effect) or by a wider practice of protective ventilation than that previously reported in braininjured patients, which may have decreased the power of the BI-VILI study to demonstrate an effect in the entire population. Fourth, the decisions to withdraw care in $22 \%$ of the population could have impacted the results. However, most of these decisions were taken after deviation from the recommendations and could be considered as outcomes rather than potential confounders. Finally, we included brain-injured patients with unaffected lungs at the time of admission. We are thus describing the effects of recommendations of protective ventilation and early extubation as a preventive strategy in patients with healthy lungs.

In conclusion, implementation of a nation-wide quality project promoting lung protective ventilation and systemic approach to early extubation did not result in a significant improvement in liberating brain-injured patients from mechanical ventilation. However, adherence to the entire set of recommendations was independently associated with improved outcomes, and neurological tolerance was good. The results indicate that lung-protective ventilation and of the early extubation in brain-injured patients could hasten weaning from invasive ventilation in brain-injured patients, although the implementation of this multi-faceted approach should be monitored and its application promoted.

\section{Electronic supplementary material}

The online version of this article (doi:10.1007/s00134-017-4764-6) contains supplementary material, which is available to authorized users.

\section{Author details}

${ }^{1}$ Intensive Care Unit, Anesthesia and Critical Care Department, Hôtel Dieu-HME-University Hospitalof Nantes, Centre Hospitalier Universitaire (CHU) de Nantes, Nantes, France. ${ }^{2}$ Department of Anesthesiology and Critical Care Department, University Hospital of Toulouse, Toulouse, France. ${ }^{3}$ Intensive Care Unit, Anesthesia and Critical Care Department, Gui Chauliac University Hospital of Montpellier, Montpellier, France. ${ }^{4}$ Intensive Care Unit, Anesthesia and Critical Care Department, Pontchaillou-University Hospital of Rennes, Rennes, France. ${ }^{5}$ Neuro-Intensive Care Unit, Anesthesia and Critical Care Department, University Hospital of Poitiers, Poitiers, France. ${ }^{6}$ Intensive Care Unit, Anesthesia and Critical Care Department, University Hospital of Angers, Angers, France. ${ }^{7}$ Intensive Care Unit, Anesthesia and Critical Care Department, University Hospital of Tours, Tours, France. ${ }^{8}$ Intensive Care Unit, Anesthesia and Critical Care Department, University Hospital of Créteil-CHU Henri Mondor, Assistance publique-Hôpitaux de Paris (AP-HP), Créteil, France. ${ }^{9}$ Department of Anesthesiology and Intensive Care, University Hospital of Clermont Ferrand, Clermont Ferrand, France. ${ }^{10}$ Department of Anesthesiology and Intensive Care, University Hospital of Nimes, Nimes, France. ${ }^{11}$ Department of Anesthesiology and Intensive Care, University Hospital of Caen, Caen, France. ${ }^{12}$ Department of Anesthesiology and Intensive Care, University Hospital of Marseille, Marseille, France. ${ }^{13}$ Intensive Care Unit, Anesthesia and Critical Care Department, Laennec-University Hospital of Nantes, Nantes, France. ${ }^{14}$ Surgical Intensive Care Unit, Anesthesia and Critical Care Department, University Hospital of Poitiers, Poitiers, France. ${ }^{15}$ Intensive Care Unit, Pasteur 2-University Hospital of Nice, Nice, France. ${ }^{16}$ Department of Surgical Sciences and Integrated Diagnostics, IRCCS AOU San Martino-IST National Cancer Research Institute, University of Genoa, Genoa, Italy. ${ }^{17}$ Intensive Care Unit, Anesthesia and Critical Care Department, Saint Eloi University Hospital of Montpellier, Montpellier, France. ${ }^{18}$ Plateforme de Biométrie, Département Promotion de la Recherche Clinique, University Hospital of Nantes, Nantes, France. ${ }^{19}$ EA 4275, MethodS for Patients-centered Outcomes and HEalth REsearch (SPHERE), UFR des Sciences Pharmaceutiques, Nantes University, Nantes, France. ${ }^{20}$ Service d'Anesthésie Réanimation, CHU de Nantes, 1 Place Alexis Ricordeau, 44093 Nantes Cedex 1, France.

\section{Acknowledgements}

The authors thank Delphine Flattres Duchaussoy, Sabrina Lebouter-Banon and the nurses of the participating ICUs for technical support.

\section{BI-VILI study group}

Dr. L. Thioliere L, Angers University Hospital; Dr. G. Zamparini G, Caen University Hospital; Dr. M. Leone, Marseille University Hospital; Drs. P.J. Mahe, D. Demeure dit latte and A. Delater, Nantes University Hospital, Hôtel Dieu; Drs. K. Lakhal K and L. Brisard, Nantes University Hospital, HGRL; Drs. R. Drilleau and O. Mimoz, Poitiers University Hospital; Drs. Y. Malledant, S. Isslame, Y. Launey and N. Nesseler, Rennes University Hospital; Dr. M. Ferrandière. Tours University Hospital.

\section{Author contributions}

RA was the principle investigator who oversaw the study conduct, helped develop all study materials including the trial protocol, assisted with participant recruitment and data collection at the Nantes site, participated in data analysis and interpretation of the results and drafted and revised the manuscript. AK provided oversight on trial design, helped develop study materials including the trial protocol, provided oversight on trial conduct, participated in data analysis and the interpretation of the results and revised the manuscript. FF, DM and SV were the trial statisticians and provided advice and input related to all statistical issues, completed final data analysis and interpretation of the results and revised the manuscript. MS, PFP, SP, DFC, LS, PA, MM, $C R, M L, H J L, H E, R B, K T, I C, C R, G T$ and $L M$ were the principal investigators at research sites and assisted with the development of the protocol and other study materials, referred or actively recruited participants at sites, assessed participant eligibility and delivered formal training. JS provided oversight on trial design, participated in data analysis and the interpretation of the results and revised the manuscript. FFV, LM, JPE and PP participated in the realisation of the meta-analysis and in the interpretation of the results and revised the manuscript.

\section{Compliance with ethical standards}

Conflicts of interest

The authors declare that they have no conflict of interest.

\section{Funding}

Non-profit organization "Gueules Cassées". 
19. The Acute Respiratory Distress Syndrome Network (2000) Ventilation with lower tidal volumes as compared with traditional tidal volumes for acute lung injury and the acute respiratory distress syndrome. N Engl J Med 342:1301-1308. doi:10.1056/NEJM200005043421801

20. Serpa Neto A, Hemmes SNT, Barbas CSV et al (2014) Incidence of mortality and morbidity related to postoperative lung injury in patients who have undergone abdominal or thoracic surgery: a systematic review and meta-analysis. Lancet Respir Med 2:1007-1015. doi:10.1016/ S2213-2600(14)70228-0

21. Amato MBP, Meade MO, Slutsky AS et al (2015) Driving pressure and survival in the acute respiratory distress syndrome. N Engl J Med 372:747755. doi:10.1056/NEJMsa1410639

22. Mascia L, Zavala E, Bosma K et al (2007) High tidal volume is associated with the development of acute lung injury after severe brain injury: an international observational study. Crit Care Med 35:1815-1820. doi:10.1097/01.CCM.0000275269.77467.DF

23. Marhong JD, Ferguson ND, Singh JM (2014) Ventilation practices in subarachnoid hemorrhage: a cohort study exploring the use of lung protective ventilation. Neurocrit Care 21:178-185. doi:10.1007/ s12028-014-0014-8

24. Elmer J, Hou P, Wilcox SR et al (2013) Acute respiratory distress syndrome after spontaneous intracerebral hemorrhage. Crit Care Med 41:19922001. doi:10.1097/CCM.0b013e31828a3f4d

25. Chesnut RM, Marshall LF, Klauber MR et al (1993) The role of secondary brain injury in determining outcome from severe head injury. J Trauma 34:216-222

26. Briel M, Meade M, Mercat A et al (2010) Higher vs lower positive end-expiratory pressure in patients with acute lung injury and acute respiratory distress syndrome: systematic review and meta-analysis. JAMA 303:865-873. doi:10.1001/jama.2010.218

27. Manzano F, Fernández-Mondéjar E, Colmenero M et al (2008) Positiveend expiratory pressure reduces incidence of ventilator-associated pneumonia in nonhypoxemic patients. Crit Care Med 36:2225-2231. doi:10.1097/CCM.0b013e31817b8a92

28. Futier $\mathrm{E}_{s}$ Constantin J-M, Paugam-Burtz C et al (2013) A trial of intraoperative low-tidal-volume ventilation in abdominal surgery. N Engl J Med 369:428-437. doi:10.1056/NEJMoa1301082

29. PROVE Network Investigators for the Clinical Trial Network of the European Society of Anaesthesiology, Hemmes SNT, Gama de Abreu M et al (2014) High versus low positive end-expiratory pressure during general anaesthesia for open abdominal surgery (PROVHILO trial): a multicentre randomised controlled trial. Lancet 384:495-503. doi:10.1016/ S0140-6736(14)60416-5

30. Mascia L, Grasso S, Fiore T et al (2005) Cerebro-pulmonary interactions during the application of low levels of positive end-expiratory pressure. Intensive Care Med 31:373-379. doi:10.1007/s00134-004-2491-2

31. Epstein SK (2002) Decision to extubate. Intensive Care Med 28:535-546. doi:10.1007/500134-002-1268-8

32. Godet T, Chabanne R, Marin J et al (2017) Extubation failure in braininjured patients: risk factors and development of a prediction score in a preliminary prospective cohort study. Anesthesiology 126:104-114. doi:10.1097/ALN.0000000000001379

33. Ferrer R, Artigas A, Levy MM et al (2008) Improvement in process of care and outcome after a multicenter severe sepsis educational program in Spain. JAMA 299:2294-2303. doi:10.1001/jama.299.19.2294

34. Sierra R, Benítez E, León C, Rello J (2005) Prevention and diagnosis of ventilator-associated pneumonia: a survey on current practices in Southern Spanish ICUs. Chest 128:1667-1673. doi:10.1378/ chest.128.3.1667

35. Bellani G, Laffey JG, Pham T et al (2016) Epidemiology, patterns of care, and mortality for patients with acute respiratory distress syndrome in intensive care units in 50 countries. JAMA 315:788-790. doi:10.1001/ jama.2016.0291

36. Zhu B, Li Z, Jiang L et al (2015) Effect of a quality improvement program on weaning from mechanical ventilation: a cluster randomized trial. Intensive Care Med 41:1781-1790. doi:10.1007/ s00134-015-3958-z

37. Rhodes A, Phillips G, Beale R et al (2015) The surviving sepsis campaign bundles and outcome: results from the International Multicentre Prevalence Study on Sepsis (the IMPreSS study). Intensive Care Med 41:1620-1628. doi:10.1007/s00134-015-3906-y 
38. Kizer JR, Cannon CP, McCabe CH et al (1999) Trends in the use of pharmacotherapies for acute myocardial infarction among physicians who design and/or implement randomized trials versus physicians in routine clinical practice: the MILIS-TIMI experience. Multicenter investigation on limitation of infarct size. Thrombolysis in myocardial infarction. Am Heart J 137:79-92

39. Bouadma L, Mourvillier B, Deiler V et al (2010) Changes in knowledge, beliefs, and perceptions throughout a multifaceted behavioral program aimed at preventing ventilator-associated pneumonia. Intensive Care Med 36:1341-1347. doi:10.1007/s00134-010-1890-9

40. Bouadma L, Mourvillier B, Deiler V et al (2010) A multifaceted program to prevent ventilator-associated pneumonia: impact on compliance with preventive measures. Crit Care Med 38:789-796. doi:10.1097/ CCM.0b013e3181ce21af
41. Klompas M (2010) Ventilator-associated pneumonia: is zero possible? Clin Infect Dis 51:1123-1126. doi:10.1086/656738

42. Lilly CM, Cody S, Zhao Het al (2011) Hospital mortality, length of stay, and preventable complications among critically ill patients before and after tele-ICU reengineering of critical care processes. JAMA 305:2175-2183. doi:10.1001/jama.2011.697

43. Concato J, Shah N, Horwitz RI (2000) Randomized, controlled trials, observational studies, and the hierarchy of research designs. N Engl J Med 342:1887-1892. doi:10.1056/NEJM200006223422507 